01

\title{
Экспериментальное и теоретическое исследование осциллятора с соударениями
}

\section{(C) В.В. Нарожнов}

Институт прикладной математики и автоматизации филиал Федерального научного центра „Кабардино-Балкарский научный центр РАН“, 360000 Нальчик, Россия

e-mail: narojnov.victor@gmail.com

Поступило в Редакцию 15 апреля 2019 г.

В окончательной редакции 19 февраля 2020 г.

Принято к публикации 27 апреля 2020 г.

\begin{abstract}
Представлены результаты исследования колебаний нелинейного механического осциллятора с упругими соударениями. Эксперимент проводился с помощью электромеханического осциллятора с соударениями. Теоретическая модель построена на основе уравнения движения с учетом упругой силы, рассчитываемой в рамках контактной теории Герца. Показано, что для осциллятора с соударениями могут иметь место бифуркации и аттракторы типа „устойчивый фокус“ и „предельный цикл“. Для анализа спектральных характеристик сигналов использовалась фурье-фильтрация.
\end{abstract}

Ключевые слова: нелинейный осциллятор, упругий удар, контактная теория Герца, бифуркации при соударениях, фурье-фильтрация.

DOI: 10.21883/JTF.2020.10.49798.166-19

\section{Введение}

Осциллятор с соударениями, являясь модельной физической системой, встречается в различных приборах и устройствах, например, в атомно-силовых микроскопах (АСМ) и микроэлектромеханических системах (МЭМС). Колебания с соударениями наблюдаются также и во многих естественных процессах, в частности в микробиологических системах.

В [1] проводилось экспериментальное и численное исследование динамики осциллятора с соударениями при наличии вязкого демпфирования. Исследовались такие характеристики, как „сила-отклонение“ и „сила-скорость“. Система изучалась с учетом нелинейной жесткости и эффектов трения, рассматриваемых отдельно. Авторы наблюдали множество бифуркационных явлений, в том числе возникновение бифуркаций при ударе (в английской терминологии - grazing bifurcations). Было отмечено, что бассейны притяжения аттрактора дают информацию относительно полного набора решений для системы, учитывая определенный набор параметров. В [2] обнаружили три основных типа бифуркаций при ударе осциллятора: 1) переход с одного устойчивого цикла на другой с добавлением каскада, 2) переход от устойчивого цикла к хаотическому аттрактору, 3) столкновение хаотического аттрактора и устойчивого цикла, который считается локальной бифуркацией. Бифуркации такого типа являются „нетрадиционными“, в том смысле, что они не встречаются в гладких системах. Помимо этого зафиксированы различные бифуркационные сценарии, возникающие при изменении амплитуды возбуждения осциллятора [3]. При этом наиболее типичный сценарий имеет место, когда устойчивый цикл аттрактора в результате соударений осциллятора переходит к другому устойчивому циклу. Другой динамический сценарий связан с эволюцией аттрактора, которая определяется сложным взаимодействием между гладкими и негладкими бифуркациями. В некоторых случаях проявилось сосуществование аттракторов через прерывистый переход от одного цикла к другому. Наблюдался также переход и к хаотическому аттрактору через удвоение цикла.

Осциллятор с соударениями можно рассматривать как частный случай билинейного осциллятора с бесконечной жесткостью при сжатии [4]. Было показано, что без учета внешней силы имеется некоторое множество собственных времен удара осциллятора, которое в целом устойчиво относительно изменения начальных условий. Если период внешней силы соответствует данному множеству собственных времен ударов, то может возникать резонансный режим колебаний. Другие режимы вынужденных колебаний не приводят к явлению резонанса. Из этого следует важный вывод: если период внешней силы соизмерим с временем между двумя последовательными ударами, то резонансный режим в данном случае может не достигаться.

В [5] изучался нелинейный динамический отклик кантилеверов АСМ при соударении зонда с образцом. Были выявлены нелинейные особенности системы сканирования АCM, включая явление многократного отскока кантилевера, ведущее к образованию петли гистерезиса. Установлена тесная связь между особенностями потенциального взаимодействия и нелинейным откликом кантилевера. В частности, подробно обсуждаются эффекты сил Ван-дер-Ваальса, наноразмерные контактные нелинейности, затухание кантилевера, а также влияние 
вынужденного и параметрического возбуждения на бифуркации и неустойчивости вынужденного периодического движения системы. Результаты показывают, что методы идентификации нелинейных систем могут быть использованы в качестве эффективных инструментов для извлечения подробной информации о потенциале взаимодействия „зонд-поверхность“. Измерения максимальной силы взаимодействия в режиме амплитудной модуляции АСМ проведены в [6]. Была также выведена формула для максимальной силы при отталкивающем гармоническом законе взаимодействия и проведено численное моделирование. Результаты численного моделирования согласуются с теорией. Кроме того, проанализированы основные факторы, влияющие на результаты эксперимента: сила адгезии, точность определения добротности кантилевера, точность измерения статической жесткости, возможное несовпадение его статической и динамической жесткости. По мнению авторов, результаты работы можно использовать для прогнозирования максимальной силы в системе зонд-образец.

Колебания с соударениями встречаются также в микромеханических и микро-электромеханических системах. Так, в [7] изучалась динамика осцилляторов c соударениями с применением кусочно-нелинейной модели. Авторы рассмотрели случай, когда деформация при соударениях велика и функционирование МЭМС происходит в области нелинейного частотного отклика. С помощью численного эксперимента было показано, что нелинейность не только сдвигает область бифуркации, но также изменяет ширину полосы пропускания. В [8] разработан и изготовлен другой вариант МЭМС - автономный механический осциллятор-ударник с МЭМС-резонатором в качестве элемента управления частотой. С помощью численного моделирования было показано, что нелинейные свойства осциллятора-ударника существенно влияют на принцип работы МЭМС. Нелинейность, в частности, проявляется в том, что амплитуда вынужденных колебаний осциллятора может изменять частоту колебаний.

Учитывая актуальность проведенных ранее исследований [1-8], целью настоящей работы являлось детальное экспериментальное и теоретическое изучение нелинейных физических явлений, возникающих при колебаниях осциллятора с соударениями. Данная цель достигалась решением следующих задач: экспериментальные исследования сигналов, возникающих при соударениях электромеханического осциллятора с поверхностью твердого тела; разработка и реализация математической модели осциллятора с соударениями; применение фурьефильтрации для интерпретации экспериментальных и теоретических сигналов.

\section{1. Эксперимент}

В настоящей работе использовался электромеханический осциллятор, конструкция которого была предло-

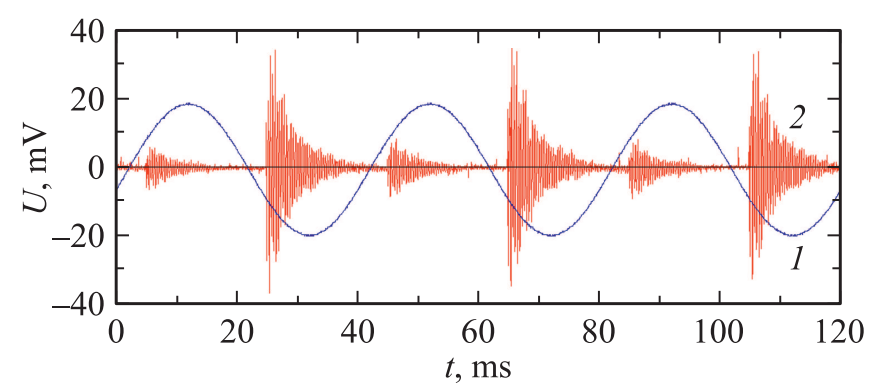

Рис. 1. Экспериментальная осциллограмма осциллятора с соударениями: 1 - сигнал возбуждения, 2 - сигнал, соответствующий соударениям осциллятора.

жена в [9]. Его принцип работы заключается в следующем. Переменный сигнал синусоидальной формы с генератора подается на катушку. Переменное магнитное поле, возникающее в катушке, приводит в колебательное движение упругий элемент (осциллятор), который представляет собой тонкую металлическую балку с корундовым зондом на свободном конце. В процессе колебаний зонд совершает удары по поверхности образца. Амплитуда колебаний осциллятора и соответственно сила удара зависят от величины переменного напряжения на катушке. Для измерения сигналов использовался пьезоэлектрический датчик и двухканальный осциллограф. Отметим, что похожие измерения с пружинным маятником проводились в [10].

В процессе эксперимента регистрировались поверхностные акустические волны с вертикальной поляризацией (так называемые волны Релея), которые возникают при ударе зонда о поверхность образца. Если упругая среда является линейной и непоглощающей, то амплитуда регистрируемых колебаний должна однозначно определяться силой удара твердого зонда о поверхность образца. В качестве образца использовалась толстая алюминиевая пластина. Модуль упругости корунда, из которого изготовлен зонд, составляет $382 \mathrm{GPa}$, что намного превышает модуль упругости алюминия $70 \mathrm{GPa}$ (указанные значения приведены в [11]). В связи с этим деформация зонда в экспериментах была исключена. Радиус кривизны кончика зонда составлял приблизительно $100 \mu \mathrm{m}$. Расстояние между зондом и пьезоэлектрическим датчиком составляло $0.5 \mathrm{~cm}$. Частота возбуждающего сигнала $25 \mathrm{~Hz}$.

На рис. 1 показаны осциллограммы сигналов: 1 сигнал, подаваемый на катушку, 2 - сигнал отклика. При максимумах и минимумах задающего сигнала 1 происходят смещения осциллятора. В промежутках между ними осциллятор совершает затухающие колебания. При этом зонд наносит удары по образцу с частотой, близкой к некоторой собственной частоте.

В экспериментах было обнаружено существенное увеличение частоты колебаний осциллятора с соударениями по сравнению с частотой задающего сигнала. Из осциллограммы на рис. 1 следует, что при частоте 
задающего сигнала $25 \mathrm{~Hz}$ частота соударений осциллятора составляет $\sim 2.5 \mathrm{kHz}$, т.е. происходит умножение частоты приблизительно в 100 раз. Тем самым осциллятор с соударениями функционирует как своеобразный электромеханический умножитель частоты.

Другое замеченное явление при малых действующих значениях переменного тока заключается в том, что пакеты сигналов, относящиеся к нарастающей и спадающей фазам задающего сигнала 1 , имеют различную амплитуду, но повторяются с каждым периодом (рис. 1). Это объясняется следующим образом. При подаче на катушку переменного тока в нарастающей фазе напряжение возрастает и магнитное поле катушки увеличивается. ЭДС самоиндукции в этот момент препятствует протеканию тока от генератора. За спадающую фазу задающего сигнала, когда напряжение на катушке уменьшается, магнитное поле создает ЭДС самоиндукции, которая совпадает по фазе с током. Ток самоиндукции в катушке старается теперь „поддержать“ уменьшающийся переменный ток и сохранить большую величину магнитного потока. В данный полупериод индуктивная катушка не потребляет энергию, а отдает ее обратно генератору. Таким образом, происходит непрерывный обмен энергий между катушкой и генератором. При этом средняя потребляемая мощность катушки равна нулю. С увеличением действующего значения переменного тока описанное явление перестает быть заметным.

\section{2. Теоретическая модель}

Для интерпретации экспериментов воспользуемся моделью, согласно которой при ударах зонда по поверхности твердого тела возникает ее упругая деформация. В теории контактного взаимодействия Герца этой деформации соответствует сила [12]:

$$
\begin{gathered}
F=-\theta(x-h) \frac{4 E \sqrt{R(x-h)^{3}}}{3\left(1-v^{2}\right)}, \\
\theta(x)= \begin{cases}1, & x \geq 0, \\
0, & x<0,\end{cases}
\end{gathered}
$$

где $\theta(x)$ - функция Хэвисайда, $x$ - смещение зонда, $h$ - высота, на которой находится зонд в начальный момент времени, $R$ - радиус кривизны кончика зонда, $E$ и $v$ - модуль упругости и коэффициент Пуассона материала образца. Функция Хэвисайда $\theta(x)$ определяет условие удара: если $x>h$, то в нижней фазе движения зонда происходит удар, в противном случае $F=0$ и деформации поверхности нет.

С учетом (1) движение осциллятора с соударениями можно описать следующей задачей Коши:

$$
\frac{d^{2} u}{d \xi^{2}}+2 a \frac{d u}{d \xi}+u+b \theta(u-1)(u-1)^{3 / 2}=c \cos \Omega \xi
$$

$$
\begin{gathered}
u(0)=0, \quad\left(\frac{d u}{d \xi}\right)_{\xi=0}=0, \\
u=\frac{x}{h}, \quad \xi=\omega_{0} t, \\
a=\frac{\gamma}{m \omega_{0}}, \quad b=\frac{4 E \sqrt{R h}}{3\left(1-v^{2}\right) m \omega_{0}^{2}}, \quad c=\frac{F_{0}}{m \omega_{0}^{2} h}, \quad \Omega=\frac{\omega_{g}}{\omega_{0}},
\end{gathered}
$$

где $\omega_{0}-$ собственная (резонансная) частота свободных колебаний осциллятора, $t-$ время, $\gamma-$ коэффициент затухания, $m$ - эффективная масса осциллятора, $F_{0}$ амплитуда внешней силы, $\omega_{g}$ - частота внешнего возбуждения, $a, b, c, \Omega-$ безразмерные коэффициенты. Коэффициент а характеризует затухание колебаний осциллятора; $b$ определяется упругими характеристиками образца; $c$ задает амплитуду приложенной внешней силы; $\Omega$ определяет частоту внешнего возбуждения. Уравнение, близкое по виду к (2), использовалось в [13] для расчета фазовых траекторий.

Дифференциальное уравнение (2) является нелинейным и получить его общее решение в аналитическом виде не представляется возможным. Задача Коши (2), (3) решалась численно. Если в данной задаче шаг интегрирования превышает характерный временной масштаб осцилляций, относящихся к соударениям осциллятора, то решение не будет устойчивым. Поэтому шаг интегрирования уменьшался до стабилизации решения с наперед заданной точностью. Кроме того, для сравнения применялся метод интегрирования Адамса. Было обнаружено, что оба метода применительно к рассматриваемой задаче дают приблизительно одинаковые результаты. Таким образом, были получены устойчивые решения задачи.
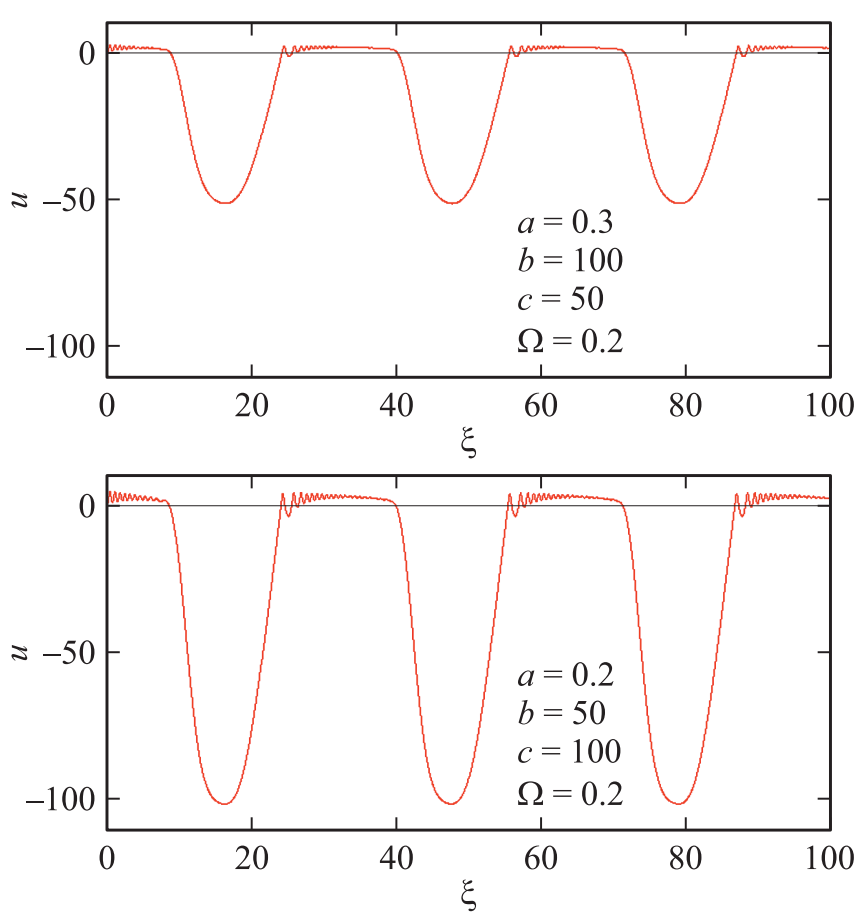

Рис. 2. Результаты численного решения задач (2), (3). 
На рис. 2 показаны примеры решений, полученные методом Рунге-Кутты 4-го порядка точности с фиксированным шагом для различных значений безразмерных коэффициентов.

Результаты численного моделирования подтверждают явление многократного отскока зонда, которое обнаруживается экспериментально. В решении, которое имеет общий осциллирующий характер, можно выделить две компоненты: низкочастотные колебания, отвечающие за приложенную внешнюю силу, и высокочастотные затухающие колебания, соответствующие ударам осциллятора. При увеличении параметра $b$ частота затухающих колебаний осциллятора возрастает, а амплитуда убывает.

На рис. 3, a представлен фазовый портрет экспериментального сигнала. Для его построения проводилась кубическая сплайн интерполяция данных с целью обеспечения непрерывности первой производной. Можно видеть, что в фазовом пространстве образуется аттрактор типа „устойчивый фокус“, который соответствует затухающим колебаниям осциллятора при его ударах о поверхность образца. Аналогичный аттрактор возникает в фазовом пространстве и при численном решении задачи (2), (3), что показано на рис. 3, $b$. Здесь также имеют место бифуркации при ударе и еще один аттрактор - „предельный цикл“, который соответствует приложенной внешней силе. В наших экспериментах данный аттрактор отсутствует, так как сигнал измеряется пьезоэлектрическим датчиком с образца. Чтобы экспериментально выявить аттрактор типа „предельный цикл“, необходимо измерять сигнал непосредственно с осциллятора.

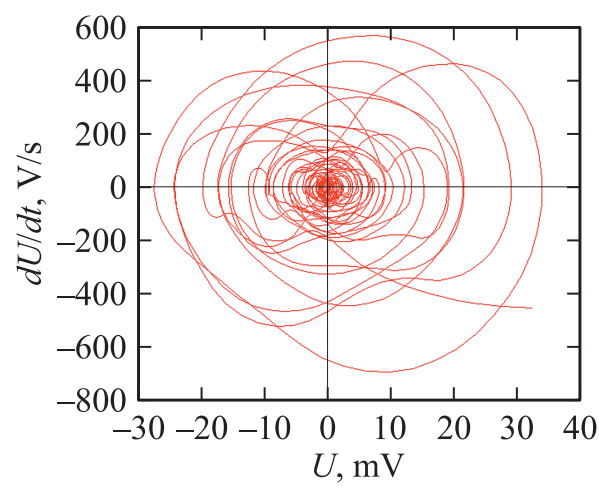

$a$

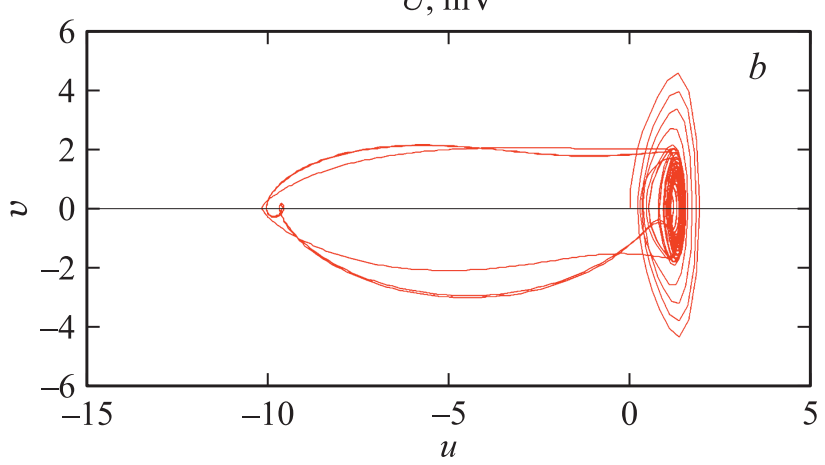

Pис. 3. Фазовые портреты: $a-$ экспериментальный сигнал, $b$ - численное решение задач (2), (3).

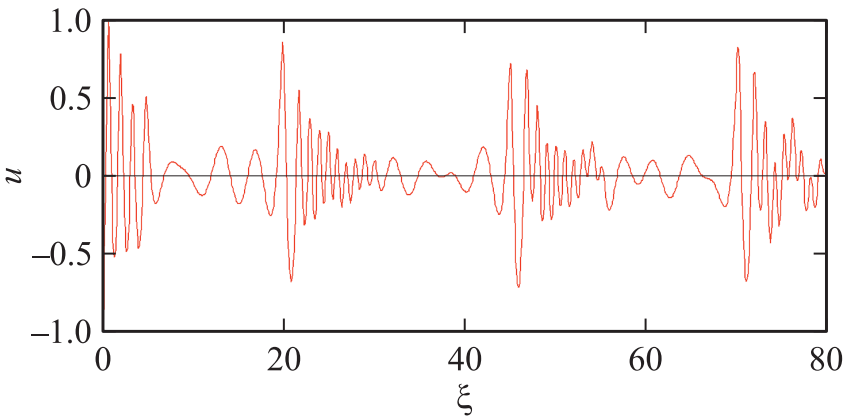

Рис. 4. Решение задач (2), (3) с выделенными частотными характеристиками.

Математическая модель (2), (3) не учитывает флуктуации, которые имеют место в натурных экспериментах. Между тем стохастический характер колебаний реального осциллятора выявляется на фазовом портрете (рис. 3,a). Несмотря на то, что колебания осциллятора описываются детерминистическим уравнением (2), в натурных экспериментах всегда обнаруживается определенный динамический хаос, который вызывается разбросом начальных условий и всевозможными механическими и электрическими шумами. Математически это может, в частности, означать, что в натурных экспериментах условия (3) не выполняются точно.

Численные решения задачи (2), (3) анализировались с применением фурье-преобразования. Проводилась фильтрация сигналов с целью выделения высокочастотных составляющих, относящихся к ударам осциллятора. Фильтрация осуществлялась путем изменения фурьеспектра

$$
C_{n}^{\prime}=C_{n} \theta\left(\Delta-\left|C_{n}\right|\right),
$$

где $\Delta$ - задает уровень обрезания спектра, $C_{n}-$ коэффициенты дискретного преобразования Фурье функции $u(\xi)$. Сигнал восстанавливается путем обратного преобразования с коэффициентами $C_{n}^{\prime}$. Результаты решения задачи фильтрации показаны на рис. 4. Здесь можно видеть, что в восстановленном сигнале отсутствуют низкочастотные колебания, соответствующие внешней переменной силе. Несмотря на наличие явления Гиббса, модельные сигналы с выделенными частотными характеристиками качественно похожи на экспериментальные (рис. 1).

\section{Заключение}

В работе представлены результаты исследования вынужденных колебаний осциллятора с соударениями. Было обнаружено, что осциллятор совершает затухающие колебания с частотой, которая может намного превышать частоту внешней силы. Для интерпретации экспериментов была предложена модель, в которой удар осциллятора описывается с помощью контактной теории Герца. Выведено нелинейное уравнение движения 
осциллятора и получены его численные решения. Численное моделирование выявляет явление многократного отскока зонда, которое наблюдается экспериментально. Показано, что для экспериментальных сигналов и численных решений уравнения движения осциллятора в фазовой плоскости имеет место аттрактор типа „устойчивый фокус“, который описывает затухающие колебания. С применением фурье-фильтрации получено численное решение с выделенными частотными характеристиками, которое качественно воспроизводит экспериментальные сигналы.

Полученные результаты могут оказаться полезными при разработке новых динамических методов диагностики упругих свойств твердых тел.

\section{Конфликт интересов}

Автор заявляет, что у него нет конфликта интересов.

\section{Список литературы}

[1] Lawrence N.V., Christopher J.B. // AIP Conf. Proceed. 2000. Vol. 502. P. 469-475.

[2] Chin W., Ott E., Nusse H.E., Grebogi C. // Phys. Rev. E. 1994. Vol. 50. N 6. P. 4427-4444.

[3] Ing J., Pavlovskaia E., Wiercigroch M., Banerjee S. // Physica D. 2010. Vol. 239. P. 312-321.

[4] Dyskin A.V., Pasternak E., Pelinovsky E. // J. Sound Vibration. 2012. Vol. 331. N 12. P. 2856-2873.

[5] Lee S.I., Howell S.W., Raman A., Reifenberger R. // Phys. Rev. B. 2002. Vol. 66. P. 115409.

[6] Щербин Б.О., Анкудинов А.В., Киюч А.В., Лобода О.С. // ФТТ. 2014. Т. 56. Вып. 3. С. 516-521.

[7] Zhang W., Turner K.L. // The 13th Intern. Conf. Solid-State Sensors, Actuators and Microsystems. Digest of Technical Papers. TRANSDUCERS'05. Seoul, Korea, 2005. June 5-9. Vol. 1. P. 768-771.

[8] Wei X., Anthony C., Lowe D., Ward M. // Procedia Chemistry 1. 2009. Vol. 1. N 1. P. 855-858.

[9] Пат. РФ. № 2552600. Рехвиашвили С.Ш., Нарожннов В.В. Стенд для исследования вязкоупругих свойств металлов и сплавов с помощью зондового акустического метода.

[10] Зейликович И.С., Никитин А.В., Василевич А.Е. // ЖТФ. 2020. Т. 90. Вып. 1. С. 5-10.

[11] Физические величины: Справочник / А.П. Бабичев, Н.А. Бабушкина, А.М. Братковский и др. / под ред. И.С. Григорьева, Е.3. Мейлихова. М.: Энергоатомиздат, 1991. $1232 \mathrm{c}$.

[12] Ландау Л.Д., Лифиии Е.М. Теория упругости. М.: Физматлит, 2001. 264 с.

[13] Pust L., Peterka F. // Meccanica. 2003. Vol. 38. P. 99-116. 